\title{
Explorando a gestão de fornecedores no contexto do CMMI-DEV e metodologias ágeis: a identificação de um gap
}

\author{
Melquizedequi Cabral dos Santos ${ }^{1,3}$ \\ Elisiane Monteiro Soares ${ }^{2}$ \\ Sandro Ronaldo Bezerra Oliveira ${ }^{2}$ \\ Alexandre Marcos Lins de Vasconcelos ${ }^{3}$
}

\begin{abstract}
Resumo: A subcontratação de software é um processo de aquisição que significa delegar, por meio de contrato, o desenvolvimento de parte, ou totalidade do produto junto a fornecedores externos ao projeto. Vários estudos obtiveram sucesso com o uso da subcontratação nesse contexto. No entanto, as organizações continuam com problemas no planejamento, execução e gestão dos fornecedores dentro do processo de aquisição no desenvolvimento de software. Este estudo conduziu uma revisão sistemática da literatura com o intuito de explorar o atual estado da investigação sobre a relação entre Metodologias Ágeis e CMMI-DEV, no contexto da gestão do acordo de fornecedores. A revisão revelou a escassez de dados empíricos sobre a temática, apontando a necessidade de mais pesquisas sobre a combinação dessas abordagens.
\end{abstract}

Palavras-chave: Gestão de Fornecedores. Melhoria de Processo de Software. Metodologias Ágeis.

\begin{abstract}
Software subcontracting is an acquisition process that means delegating, through a contract, the development of part or the entire product with external suppliers to the project. Several studies have been successful with the use of subcontracting in this context. However, organizations continue having problems in the planning, execution and management of suppliers within the acquisition process in software development. This study led to a systematic review of the literature in order to explore the current state of research on the relationship between Agile Methodologies and CMMI-DEV, in the context of supplier agreement management. The review revealed the scarcity of empirical data on the subject, pointing out the need for more research on the combination of these approaches.
\end{abstract}

Keywords: Suppliers Management. Software Process Improvement. Agile Methodologies.

\section{Introdução}

Desenvolver software de qualidade, dentro do escopo, tempo, custo e com os riscos controlados é um dos grandes desafios das organizações [1, 2, 3]. No intuito de mitigar esses desafios, as empresas de desenvolvimento de software estão buscando novas estratégias como a subcontratação de parte dos produtos e/ou serviços oferecidos aos clientes na tentativa de atender o mercado dinâmico e melhorar a qualidade do processo e do produto final $[1,2,4]$. Por exemplo, a estratégia de subcontratação de software é um processo de aquisição que significa delegar, por meio de contrato formal, o desenvolvimento de parte, ou totalidade do produto junto a fornecedores externos ao projeto. Após a formalização contratual, estes fornecedores juntam-se à estratégia organizacional do contratante na busca pela solução dos problemas dos clientes $[1,2,4]$.

\footnotetext{
${ }^{1}$ Instituto Federal do Sertão Pernambucano - IFSERTÃO-PE, Petrolina (PE) - Brasil

\{mcs6ecin.ufpe.br\}

${ }^{2}$ Programa de Pós-Graduação em Ciência da Computação da Universidade Federal do Pará, UFPA, Belém (PA) - Brasil \{elismclean@gmail.com, srbo@ufpa.br

${ }^{3}$ Centro de Informática da Universidade Federal de Pernambuco, CIN UFPE, Recife (PE) - Brasil

$\{$ amlv@cin.ufpe.br\}
}

http://dx.doi.org/10.5335/rbca.v9i2.6042 
Várias experiências de sucesso na utilização da estratégia de subcontratação têm sido publicadas $[5,6,7$, $8,9,10,11,12]$. Essas publicações, também, apontam que as organizações continuam com problemas no planejamento, execução e gestão dos fornecedores dentro do processo de aquisição no desenvolvimento de software. Estudos mostram a dificuldade em manter o controle sobre o processo de desenvolvimento desses fornecedores, problemas no gerenciamento da qualidade dos produtos e serviços adquiridos, bem como, planejamento precário, entrega feita em atraso e custos não planejados $[4,5,6,7,10,13,14,15]$. Visando resolver os problemas, as empresas estão adotando cada vez mais modelos de qualidade e metodologias de desenvolvimento de software com foco na melhoria do processo de software e na qualidade do produto [1,2]. $\mathrm{O}$ CMMI-DEV (Capability Maturity Model Integration for Development) possui, entre outras, uma área de processo chamada de Gestão de Acordos com Fornecedores (SAM - Supplier Agreement Management), cujo objetivo é gerir a aquisição de produtos de fornecedores através de um contrato formal [16].

Neste mesmo contexto, as metodologias ágeis compartilham princípios como: a satisfação do cliente, valorização da equipe, colaboração, motivação, entre outros. Esses princípios podem ser alcançados através de práticas ágeis como: ciclos iterativos, entregas antecipadas e contínuas de software funcional, simplicidade no desenvolvimento das atividades, entre outras [17, 18]. De acordo com Sommerville [3] e Dybå e Dingsøyr [19], atualmente existem diversas metodologias que buscam seguir os princípios ágeis, porém, as mais conhecidas, segundo levantamento feito [20, 21, 22, 23], são Scrum [24], XP (eXtreme Programming) [25, 26], Crystal [27], Lean[28], FDD (Feature Driven Development) [29] e DSDM (Dynamic Systems Development Method) [30].

Vários estudos relatam os benefícios do uso das metodologias ágeis no desenvolvimento de software, como: melhoria na comunicação da equipe do projeto e feedback rápido e frequente [31]; melhora no monitoramento e controle do projeto [32]; maior colaboração entre a equipe do projeto [33]; entrega frequente de software funcional [34]; facilita a resolução de problemas [35]; impactos positivos na satisfação dos stakeholders[36]; entre outros. Diante do exposto, acredita-se ser viável a utilização dos princípios e práticas ágeis para resolver os problemas mencionados. Acredita-se que, por similaridade, os benefícios alcançados com o uso dessas metodologias em outras situações no desenvolvimento de software também podem ser alcançados dentro do processo de aquisição e consequentemente melhorar a qualidade da gestão dos fornecedores no desenvolvimento de software.

Nesse contexto, aparece o seguinte questionamento: como é possível resolver os problemas da gestão de fornecedores no desenvolvimento de software a partir da combinação das práticas de SAM do CMMI-DEV com os princípios e práticas ágeis? Este estudo parte da hipótese que um processo híbrido, ao aliar as práticas de SAM do CMMI-DEV com os princípios e práticas ágeis, melhora a qualidade da gestão dos fornecedores no desenvolvimento de software. No entanto, ainda são escassas as evidências sobre como as metodologias ágeis e a gestão de fornecedores está sendo utilizadas em ambientes reais e quais são os seus impactos $[19,37,38,39,40]$.

Esta pesquisa está sendo desenvolvida no contexto de uma tese de doutorado. Então, antes de executar métodos experimentais, como: estudo de caso, pesquisa-ação, experimento controlado, entre outros [41]; para responder a questão de pesquisa e comprovar ou refutar a hipótese, foi necessário verificar se estas já não haviam sido respondidas ou comprovadas em outros estudos. Dessa forma, o objetivo inicial deste estudo foi explorar a literatura, através de um Estudo de Revisão Sistemática da Literatura (RSL) [42], para analisar o atual estado da investigação sobre a relação entre Metodologias Ágeis e CMMI-DEV, no contexto da gestão do acordo de fornecedores, em resposta à questão de pesquisa.

A revisão mostrou a escassez de dados empíricos sobre a temática, apontando a necessidade de mais pesquisas sobre a combinação das práticas da área de processo SAM do CMMI-DEV com as metodologias ágeis. Dessa forma, confirmam a lacuna de pesquisa na área investigada, justificando a relevância, viabilidade e o caráter inovador desta pesquisa. A principal contribuição deste estudo foi à identificação de um GAP com novas perspectivas e lacunas de pesquisa sobre o tema que podem ser exploradas em estudos futuros através de estudos experimentais, os quais poderão promover uma melhoria na área investigada. Os resultados dessa pesquisa também irão contribuir para a produção de conhecimento científico que promova o desenvolvimento e a melhoria do processo de gestão do acordo de fornecedores no contexto dos métodos ágeis e do CMMI-DEV.

As próximas seções descrevem: a revisão da literatura e trabalhos relacionados na Seção 2; na Seção 3 é apresentada a metodologia da pesquisa; na Seção 4 são apresentados os resultados, a síntese, a análise e a discussão; na Seção 5 são apresentadas as conclusões e os trabalhos futuros. 


\section{Revisão da literatura e trabalhos relacionados}

Esta seção apresenta informações gerais do estado da arte da pesquisa, bem como uma análise dos trabalhos relacionados.

\subsection{Aquisição de software}

A terceirização de software em Tecnologia da Informação sofreu uma grande transformação na última década, pois obteve considerável aumento na quantidade de aquisição de software, realizada sob demanda [43]. Os acordos entre as partes envolvidas têm evoluído para operações complexas abrangendo vários sistemas e processos que estão sujeitos às regulamentações de acordo com cada país fornecedor de produtos de software. Por esse motivo, o procedimento de aquisição de software deve ser tratado como um projeto e, como tal, precisa ser devidamente gerenciado de forma mais ágil para que consiga atender às demandas que o mercado exigir.

Para Nunes [44], a aquisição de software e a gestão dos projetos adquiridos têm sido estudadas em diversos países e existem modelos que tratam do desenvolvimento de software com aquisição por meio de terceiros, como os descritos no CMMI. Este modelo foi criado pelo SEI - Software Engineering Institute em parceria com a General Motors e possui dois modelos relacionados ao processo de aquisição: o modelo CMMIACQ - CMMI for Acquisition[1] que possui as boas práticas para gerenciar a aquisição de produtos e serviços com o objetivo de atingir as necessidades do cliente, e que embora fornecedores de software possam também utilizá-lo como referência, seu foco é o adquirente; e o modelo CMMI-DEV, que deve ser utilizado por fornecedores que utilizam a aquisição de software para desenvolverem produtos e serviços.

Neste trabalho, o principal modelo estudado foi o CMMI-DEV, já que possui um processo de apoio ao gerenciamento de acordos com fornecedores (SAM) na aquisição de produtos e serviços em projetos de desenvolvimento.

\subsection{Aquisição de software no contexto CMMI-DEV v 1.3}

O CMMI-DEV é um modelo de maturidade e capacidade integrado para desenvolvimento de software voltado para melhoria da qualidade dos processos, produtos e serviços relacionados. Esse modelo envolve a gestão de projeto, gestão de processo, engenharia de software, sistemas e hardware, bem como, as práticas de suporte. Ele oferece uma oportunidade para evitar ou eliminar barreiras existentes nesse processo, sendo um modelo muito usado na medição da qualidade do processo de desenvolvimento de produto de uma organização. Consiste em 22 áreas de processo que possuem um conjunto de práticas [1].

A aquisição de software no contexto do CMMI-DEV é tratada dentro do escopo da gestão de projeto do nível 2 de Maturidade do CMMI-DEV v1.3, ou seja, a gestão da aquisição de software é realizada através de uma área de processo chamada Supplier Agreement Management ou gestão de acordos/contratos com fornecedores. O propósito dessa área é gerenciar a aquisição de parte dos produtos de software e/ou serviços junto a fornecedores externos ao projeto, em conformidade com um acordo ou contrato formal [1]. Á área de processo SAM possui dois objetivos: (i) estabelecer acordos com fornecedores; e (ii) satisfazer acordos com fornecedores. Esses objetivos podem ser alcançados por meios das seguintes práticas específicas: determinação do tipo de aquisição, seleção de fornecedores, estabelecer e manter acordos com fornecedores, execução de contratos com fornecedores, aceitação da entrega de produtos adquiridos e assegurar uma transição bem sucedida dos produtos adquiridos [1].

\subsection{Metodologias ágeis}

As metodologias ágeis de desenvolvimento de software nasceram da insatisfação com as metodologias tradicionais e da necessidade de desenvolver softwares cada vez mais adaptativos às inovações tecnológicas do mercado competitivo, bem como pela necessidade de respostas às mudanças rápidas dos requisitos de negócio, e pela pressão para entrega de software cada vez mais rápida, de qualidade e que satisfaça as necessidades dos stakeholders [3]. Em 2001, nasceu o Manifesto Ágil para o desenvolvimento de software [17,18], que define o desenvolvimento de software ágil de software em termos de valores como: (a) Indivíduos e interações mais que processos e ferramentas; (b) Software em funcionamento mais que documentação abrangente; (c) Colaboração com o cliente mais que negociação de contratos; (d) Responder a mudanças mais que seguir um plano. De 
acordo com Sommerville [3] e Dybå e Dingsøyr [19], atualmente existem diversas metodologias que buscam seguir os princípios ágeis, porém, as mais conhecidas, segundo levantamento feito pela [20,21, 22, 23], são Scrum[24], eXtreme Programming [25, 26], Crystal [27], Lean[28], Feature Driven Development [29], Dynamic Systems Development Method [30].

\subsection{Trabalhos relacionados}

Santana et al. [37] realizaram uma revisão sistemática da literatura com o objetivo de identificar e caracterizar Melhoria de Processos de Software (Software Process Improvement - SPI) em ambientes ágeis, que retornou 423 artigos publicados no período de 2001 a março/2013. Foram selecionados 31 trabalhos que discutiam Ágeis e SPI, classificados de acordo com aspectos SPI, atividades destinadas à melhoria dos processos e práticas que envolvem o desenvolvimento de software. A pesquisa permitiu a identificação de abordagens distintas para Ágil e SPI. A abordagem mais comum consiste em adaptar as abordagens tradicionais de SPI em ambientes ágeis, outras abordagens estão relacionadas com a melhoria do comportamento das equipes ou melhoria de práticas inovadoras em SPI.

Silva et al. [38] realizaram uma revisão sistemática com intuito de avaliar, sintetizar e apresentar resultados sobre o uso do modelo CMMI em combinação com desenvolvimento ágil de software, para fornecer uma visão geral dos temas pesquisados, no período de 1998 a 2011, realizando uma discussão de seus benefícios e limitações, e as implicações para a pesquisa e a prática. A pesquisa inicialmente identificou 3193 estudos, dos quais 445 foram consideradas potencialmente relevantes e 81 estudos foram incluídos, pois respondiam as questões de pesquisa do trabalho. Com base nos resultados obtidos a partir da revisão, foi proposto um conjunto de estratégias e abordagens, combinando CMMI e métodos ágeis.

Patel e Ramachandran [45] desenvolveram uma pesquisa sobre o modelo de adaptabilidade, adequação e maturidade de software, que definiu um modelo de processo genérico de melhoria do processo de software, denominado Modelo de Maturidade Ágil (AMM), adequado para ambientes de desenvolvimento de software ágeis, o qual identificou e definiu as práticas ágeis para cada nível de maturidade e relacionou os problemas de práticas ágeis com as metas de melhoria de práticas ágeis.

O estudo realizado por Santana et al. [46] analisou dados quantitativos e qualitativos recolhidos na literatura e em duas empresas brasileiras que trabalham com a metodologia ágil XP. A contribuição gerada está relacionada aos elementos relevantes sobre a associação de ágil e CMMI, bem como, sobre as consequências da aplicação desta metodologia resultante da fusão incompleta destas abordagens, uma vez que foi constatado que existem poucos relatos quantitativos, tanto para dar parecer definitivo sobre esta maneira "incompleta" de fusão, quanto para definir em que casos que a fusão poderia trazer melhores resultados.

A pesquisa desenvolvida por Jakobsen e Johnson [47] foi bem específica e mostrou como a metodologia ágil Scrum pode ser combinada com sucesso ao modelo CMMI e gerar resultados positivos para o desenvolvimento de atividades inerentes a projetos. O trabalho apresenta as experiências sobre como o CMMI pode dar suporte para práticas ágeis e recomenda um subconjunto de atividades, inspiradas nos princípios ágeis. O documento não descreve como amadurecer do CMMI nível 1 para o nível 2, mas destaca um conjunto de atividades que poderiam ser consideradas "a ponte" entre ágil, apenas para os projetos com base em Scrum e as disciplinas esperados de projetos e organizações que trabalham para o CMMI nível 2 ou 3.

Os trabalhos citados mostraram em vários contextos a possibilidade de combinação do modelo CMMI com as metodologias ágeis dentro do desenvolvimento de software. No entanto, observou-se que a gestão do acordo de fornecedores dentro do processo de aquisição de partes do produto ou serviços nesse contexto tem ficado fora do escopo dessas pesquisas. O principal diferencial desta pesquisa em relação aos trabalhos relacionados foi o desenvolvimento de uma revisão sistemática para entender o atual estado da investigação empírica acadêmica e industrial sobre as metodologias ágeis que apoiam o processo de SAM no contexto das empresas de desenvolvimento de software. 


\section{Metodologia}

Esta pesquisa conduziu uma revisão sistemática seguindo o guia estabelecido por Kitchenham e Charters [42] e Kitchenham et al. [48], por ser uma das referências mais adotadas em estudos secundários na área de engenharia de software.

\subsection{Questões de pesquisa}

Com base no objetivo de pesquisa foi definida a seguinte questão de pesquisa:

(Q1)Qual o atual estado da investigação sobre a relação entre Metodologias Ágeis e CMMI-DEV, no contexto da gestão de fornecedores? Antes de executar o MS, foi verificada na literatura se já existia alguma pesquisa secundária publicada que respondesse às questões de pesquisa elaboradas. Assim, foi realizada uma pesquisa adhoc nas bases de dados definidas no protocolo e não foram encontrados estudos secundários sobre a área de processo SAM do CMMI-DEV e Metodologias Ágeis.

\subsection{Protocolo da pesquisa}

Um processo de pesquisa amplo e revisado por pares foi conduzido em busca de estudos acadêmicos publicados em inglês e português no período de 01 janeiro de 2001 a 31 de dezembro de 2016, ou seja, 16 anos. Este estudo adotou uma abordagem de busca automática e para aumentar a cobertura da pesquisa, também foi incluída uma busca manual em eventos de relevância não indexados nas bases de dados digitais. Para aumentar a possibilidade de transferência dos resultados para outros contextos, o protocolo foi descrito em detalhes.

\subsection{Termos e string de busca}

Os termos de busca foram construídos em duas fases. A primeira, as palavras-chave foram identificadas em cada questão de pesquisa. A segunda, os sinônimos foram definidos para todas as palavras-chave. Foram realizadas várias iterações e testes pilotos com a string para garantir um conjunto abrangente. Acredita-se que foi atingido um nível de cobertura razoável com esses termos e sinônimos. Os termos de busca e seus sinônimos foram definidos em Português e traduzidos para o Inglês, para proporcionar uma maior cobertura possível sobre o tema, conforme Tabela 1 .

Tabela 1: Termos de busca

Termos de busca

Capability Maturity Model Integration, CMMI,CMMI-DEV; Agile, Scrum, extreme programming, $X P$, crystal, lean, feature driven development, FDD, Dynamic systems development method, DSDM, adaptive software development, test driven development, TDD; Supplier, SAM, acquisition, subcontracting, outsourcing; Software, system, application

Os termos da string foram unidos com o operador "OR" e o conjunto de sinônimos foram concatenados usando o operador "AND". Testes pilotos foram realizados com a string para verificar se os principais autores e artigos na área foram recuperados. Exemplo da string em inglês: ("Capability Maturity Model Integration" OR "CMMI" OR "CMMI-DEV") AND ("agile" OR "scrum" OR "extreme programming" OR "XP" OR "dynamic system development" OR "DSDM" OR "crystal" OR "feature driven development" OR "FDD" OR "lean" OR "adaptive software development" OR "ASD" OR "test driven development" OR "TDD") AND ("supplier" OR "SAM" OR "acquisition" OR "subcontracting" OR "outsourcing") AND ("software" OR "system" OR "application").

\subsection{Fontes de busca}

Esta pesquisa realizou buscas automáticas e manuais visando garantir uma maior cobertura possível da literatura sobre o tema [42]. Para as buscas automáticas foram escolhidas as seguintes bases de dados digitais que indexam as principais conferências e periódicos internacionais na área de investigação: (1) Compendex (Engineering Village), (2) Science Direct - Elsevier, (3) Scopus - Elsevier, (4) IEEE Xplore, (5) ACM Digital 
Library, (6) Portal de periódicos Capes/MEC, (7) Springer, (8) ISI Web of Knowledge, (9) Jairo, (10) Wiley Inter Science Journal.

A base de dados Google Scholar não foi incluída devido ao alto índice de duplicação em relação às fontes automáticas. Para as buscas manuais foram escolhidas as principais conferências, workshops e simpósios da área, as quais não estavam indexadas nas bases de automáticas: (1) WAMPS - Workshop Anual do MPS.BR, (2) SBES - Simpósio Brasileiro de Engenharia de Software.

\subsection{Critérios de inclusão (CI) e exclusão (CE)}

Foram incluídos os trabalhos que atenderam os seguintes critérios: CI-01-Somente estudos primários; CI02-Estudos com dados empíricos; CI-03-Estudos que não apresentam resultados de ensino do tema; CI-04Estudos relacionados à CMMI-DEV, metodologias ágeis, gerenciamento de contrato com fornecedores (SAM) e desenvolvimento de software; CI-05-Estudos completos publicados entre o período de 01/01/2001 a 31/12/2016. O período se estendeu até o ano de 2016, ano de desenvolvimento e atualização da pesquisa; CI-06-Estudos escritos em Português e Inglês. Foram excluídos os trabalhos que preencheram qualquer um dos seguintes critérios: CE-01-Estudos com relatório de experiência sem método de pesquisa definido; CE-02-Estudos que se apresentam no formato de editoriais, prefácios, correspondência, debates; CE-03-Estudos que se concentram em ensinar o tema da pesquisa; $\mathrm{CE}-04$-Estudos que não respondem as nossas perguntas de pesquisa; $\bullet$ CE-05-Estudos repetidos sem informação adicional.

\subsection{Processo de seleção dos estudos}

Esta pesquisa foi realizada em quatro fases, conforme Figura 1, sendo conduzida por dois pesquisadores e três revisores para minimizar o viés de seleção [42].

Figura 1: Fases da Pesquisa

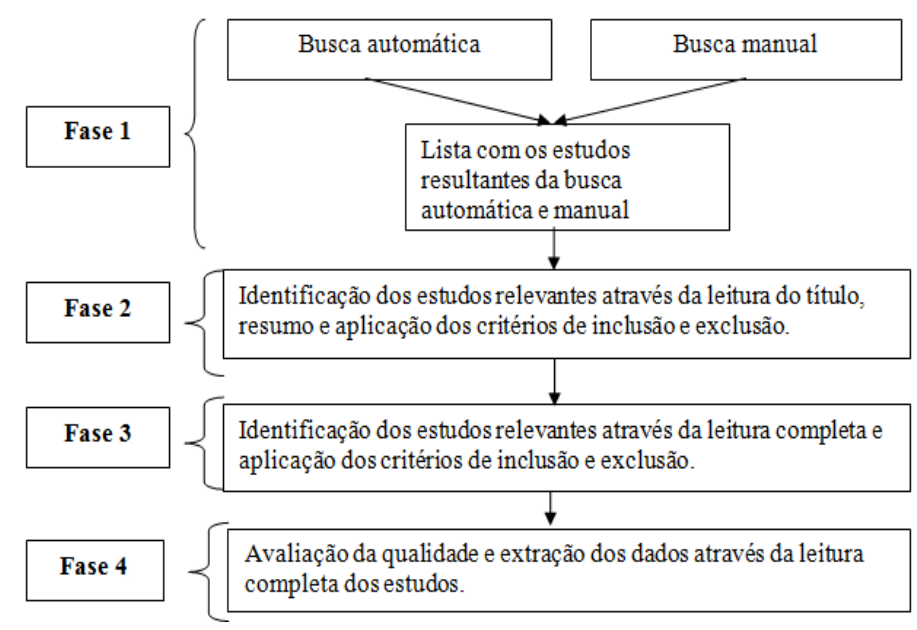

Fase 1: A busca automática constitui em acessar as fontes de busca e executar a string de pesquisa para obtenção dos artigos relevantes conforme critérios definidos no protocolo. A busca manual constitui em acessar as conferências e baixar todos os artigos relacionados ao tema de pesquisa. Os estudos encontrados foram registrados com um identificador, por exemplo: $S x x x x$, onde $S=($ estudo) e $x x x x=$ (número sequencial), e classificados em uma planilha eletrônica que foi armazenada em um repositório online e compartilhada por todos os pesquisadores. Fase 2: Cada estudo selecionado na etapa anterior foi analisado por dois pesquisadores, com base na análise do título e resumo, descartando os estudos duplicados e os que são claramente irrelevantes para a pesquisa de acordo com os critérios de inclusão e exclusão. Foi formada uma lista de estudos incluídos e excluídos por cada pesquisador da dupla. As listas foram comparadas e os conflitos foram discutidos entre os pesquisadores, quando não houve consenso sobre as divergências, os artigos em conflito foram incluídos para uma análise mais detalhada na próxima fase. Ao final desta fase, uma lista, consolidando os estudos relevantes, foi armazenada em um repositório online e compartilhada por todos os pesquisadores. Fase 3: A partir da lista 
consolidada da fase 2, todos os estudos foram avaliados por dois pesquisadores, mediante a aplicação dos critérios de inclusão e exclusão, através da leitura completa. Em caso de conflito de julgamento entre os avaliadores, foi realizada uma reunião de consenso, e na persistência destes, o conflito solucionado pelo revisor da pesquisa. Uma lista consolidada com os estudos relevantes foi armazenada em um repositório online e compartilhada por todos os pesquisadores. Fase 4: A partir da lista consolidada da fase 3 foi realizada a avaliação da qualidade e extração dos dados de todos os estudos, através da leitura completa dos estudos. Para avaliação da qualidade, adotou-se a estratégia defina na Seção 3.7. A extração de dados foi realizada por dois pesquisadores e os resultados foram documentados em formulário, sendo a revisão da extração realizada pelo revisor da pesquisa.

\subsection{Avaliação da qualidade}

Os critérios de qualidade (CQ) foram definidos baseados em Dybå e Dingsøyr [19] e Kitchenham e Charters [42], conforme Tabela2.

Tabela 2: Critérios de qualidade (CQ)

\begin{tabular}{l} 
Tabela 2: Critérios de qualidade (CQ) \\
\hline \multicolumn{1}{c}{ Critérios de Qualidade } \\
\hline CQ1-Existe uma clara declaração dos objetivos da pesquisa? \\
CQ2-Existe uma descrição adequada do contexto? \\
CQ3-O projeto de pesquisa foi adequado para abordar os objetivos? \\
CQ4-A abordagem de pesquisa está definida claramente? \\
CQ5-A estratégia de pesquisa foi adequada aos objetivos da pesquisa? \\
CQ6-Havia um grupo de controle com o qual comparar tratamentos? \\
CQ7-Os dados foram coletados abordando a questão de pesquisa? \\
CQ8-A análise dos dados foi suficientemente rigorosa? \\
CQ9-A relação entre o pesquisador e os participantes foi considerada adequadamente? \\
Reconhecimento de viés pelo pesquisador. \\
CQ10-Existe uma declaração clara dos resultados? \\
CQ11-O trabalho apresenta o valor do estudo para pesquisa ou prática?
\end{tabular}

Os pesquisadores devem avaliar os critérios de qualidade CQ1 a 11 de acordo com a escala Likert[49] de três pontos: Não Atende (0) - deve ser concedido no caso em que não existe nada no trabalho que atenda ao critério avaliado; Neutro (0.5) - deve ser atribuído no caso em que o trabalho não deixa claro se atende parcialmente ou não ao critério avaliado; Atende (1) - deve ser fornecido no caso em que o trabalho apresente claramente no texto o critério avaliado. Os desacordos devem ser resolvidos em reunião de consenso. Para cada estudo deverá ser feita uma avaliação da qualidade de forma individual pela dupla, baseada nos critérios de qualidade da Tabela 2. Os pesquisadores devem atribuir notas aos critérios, de acordo com a escala de Likert definida. Ao final será feito o somatório da pontuação e calculada a nota total do estudo em relação à qualidade. A classificação final da qualidade dos estudos será feita conforme faixas de qualidade definidas em Beechan [50], como podem ser visto na Tabela 3. Os estudos com qualidade baixa devem ser excluídos.

Tabela 3: Classificação da qualidade

\begin{tabular}{ll}
\hline Nota do Estudo (\%) & $\begin{array}{l}\text { Classificação } \\
\text { qualidade }\end{array}$ \\
\hline Nota $>=86 \%$ & Excelente \\
$66 \%=<$ Nota $<=85 \%$ & Muito Boa \\
$46 \%=<$ Nota $<=65 \%$ & Boa \\
$26 \%=<$ Nota $<=45 \%$ & Média \\
Nota $<26 \%$ & Baixa \\
\hline
\end{tabular}

\subsection{Extração, síntese e análise dos dados}

Este estudo adotou o processo de extração dos dados recomendado por Cruzes e Dyba [51], como pode ser visto na Figura 2. 
Figura 2: Estrutura de extração dos dados [51]

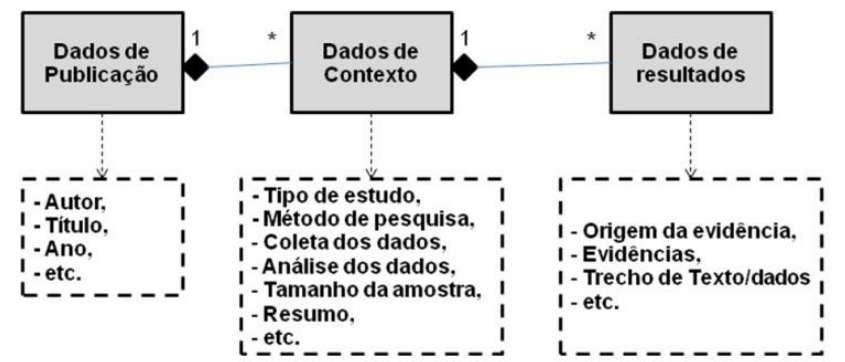

A Figura 2 mostra que a extração dos dados relevantes deve seguir uma estrutura. Tal estrutura é formada por informações de publicação, de contexto e de resultados. Os estudos que não apresentarem informações relevantes para responder as questões de pesquisa serão excluídos. Nesse sentido, a extração dos dados será realizada da seguinte forma: a) Leitura cuidadosa dos estudos, para que o pesquisador fique imerso e familiarizado com os dados; b) Identificação dos segmentos de texto relacionados com os objetivos da pesquisa; c) Extrair os dados de publicação, contexto e resultados em resposta às questões de pesquisa; d) Revisão da extração dos dados com outro pesquisador.

A síntese e análise dos dados foram construídas paralelamente baseadas em uma abordagem qualitativa e quantitativa. Assim, este estudo seguirá uma síntese e análise temática dos dados, conforme processo adaptado definido por Cruzes e Dyba [51]. Em resumo, o processo consiste em: fazer uma leitura inicial dos estudos; posteriormente, realizar a extração dos dados e evidências, identificando os códigos (textos) de dados, onde esses códigos serão traduzidos em temas/categorias e apresentados em tabelas e gráficos para explicação do fenômeno ou questões de pesquisa; planilhas eletrônicas serão utilizadas para facilitar a manipulação e apresentação dos dados; a partir da coleta dos dados serão realizadas as comparações e análises.

\section{Resultados}

Esta seção apresenta os principais resultados obtidos com a pesquisa realizada pela RSL conduzida.

\subsection{Processo de seleção dos estudos}

A Figura 3 resume as fases e o número de estudos identificados em cada etapa da RSL.

Figura 3: Resultado do Processo de Seleção dos Estudos.

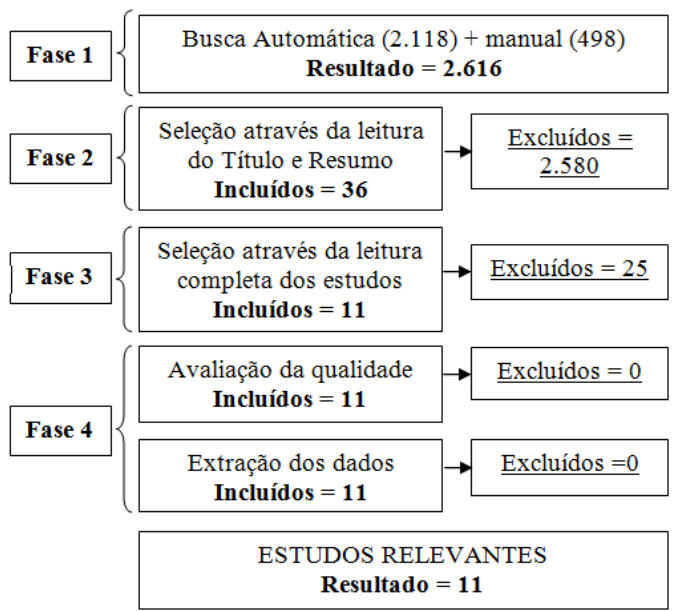

Fase 1 - Busca Automática e Manual: No início desta fase foram realizados testes pilotos nas bases de dados digitais com o intuito de adaptar a string de busca a cada repositório. Este procedimento foi uma ação no sentido de padronizar as buscas automáticas em todos os engenhos, com pequenas adaptações nas ferramentas de 
busca. Essa estratégia permitiu melhorar um contexto de replicação futura deste estudo. No entanto, a string possui termos genéricos como: CMMI-DEV, Desenvolvimento de Software e Ágil. Esses termos são comuns nos referenciais teóricos dos artigos, ocasionando um alto número de artigos, bem como duplicação e exclusões por falta de evidências. A partir da string, as buscas automáticas e manuais foram realizadas no período de 01/01/2001 a 31/12/2016. Foram identificados 2.616 estudos, sendo 2.118 resultantes das buscas automáticas realizadas em dez bases de dados eletrônicas: Compendex, com 7, cerca de 0,30\% do total; Science Direct, com 301, cerca de 11,50\%; Scopus, com 312, cerca de 11,90\%; IEEE Xplore, com 265, cerca de 10,12\%; ACM, com 87, cerca de 3,30\%; Portal de Periódicos capes, com 186, cerca de 7,11\%; Springer, com 569, cerca de 21,80\%; ISI WEB, com 95, cerca de 3,60\%; Jairo, com 0, cerca de 0,00\%; Wiley Library, com 295, cerca de 11,27\%. As Buscas manuais revelaram 498 estudos, sendo distribuídos em: WAMPS, com 124, cerca de 4,80\%; e SBES, com 374, cerca de 14,30\%. Fase 2 - Seleção dos Estudos pelo Título e Resumo: Nesta fase, os dois pesquisadores realizaram a leitura do título e resumo de todos os 2616 artigos, de forma individual e independente, aplicando os critérios de seleção e descartando os artigos duplicados e irrelevantes. Os conflitos foram discutidos em reunião de consenso pela dupla. Dessa forma, foi possível rejeitar 2580 artigos considerados irrelevantes para o escopo da pesquisa. Esta fase finalizou com a inclusão de 36 estudos potencialmente relevantes. Fase 3 - Seleção dos Estudos pela Leitura Completa: Os 36 estudos resultantes da fase anterior foram distribuídos e cada pesquisador realizou a leitura completa, de forma individual e independente, aplicando os critérios de seleção. Dessa forma, 25 estudos foram considerados irrelevantes e foram excluídos. Ao final, 11 artigos foram identificados como relevante. Fase 4 - Avaliação da Qualidade e Extração dos dados: Em relação à análise da qualidade dos estudos identificados, considerou-se a qualidade dos artigos como sendo boa, pois, levou-se em consideração que os artigos publicados nessas fontes de dados já passaram por um rigoroso processo de análise e seleção da qualidade para publicação. Em relação a extração dos dados foi feito o processo descrito no protocolo.

\subsection{Discussão dos resultados}

Ao final do processo de seleção, onze estudos relacionados ao tema foram encontrados como relevantes, pois abordaram as disciplinas CMMI, Metodologias ágeis e gestão de fornecedores. A identificação e a referência dos estudos podem ser visualizadas na Tabela 4.

Tabela 4: Identificação e referência dos estudos

\begin{tabular}{|c|c|}
\hline ID & Referência \\
\hline S0004 & $\begin{array}{l}\text { NAVARRETE, F.; BOTELLA, P.; FRANCH, X. Reconciling Agility and Discipline in COTS } \\
\text { Selection Processes. in Commercial-off-the-Shelf (COTS)-Based Software Systems. Sixth } \\
\text { International IEEE Conference, p.103-113, DOI: } 10.1109 / \text { ICCBSS.2007.29. } 2007\end{array}$ \\
\hline S0329 & $\begin{array}{l}\text { LEE, SUNG-WOOK; KIM, HAENG-KON; LEE, ROGER Y. Enterprise Process Model for } \\
\text { Extreme Programming with CMMI Framework. Computer and Information Science. 2008: 169- } \\
\text { 180. DOI 10.1007/978-3-540-79187-4_15 }\end{array}$ \\
\hline S0383 & $\begin{array}{l}\text { PIKKARAINEN, M.; MÄNTYNIEMI, A. An approach for using CMMI in agile software } \\
\text { development assessments: Experiences from three case studies. 6th Conference on Software Process } \\
\text { Improvement and Capability Determination, SPICE } 2006 \text {. }\end{array}$ \\
\hline S0449 & $\begin{array}{l}\text { MARCAL, A.S.C.; SOARES, F. S. F.; BELCHIOR, A. D. Mapping CMMI Project Management } \\
\text { Process Areas to SCRUM Practices. in Software Engineering Workshop. 2007. pp.13-22. DOI: } \\
\text { 10.1109/SEW.2007.102 }\end{array}$ \\
\hline S0468 & $\begin{array}{l}\text { SUTHERLAND, J.; JAKOBSEN, C.R.; JOHNSON, K..Scrum and CMMI Level 5: The Magic } \\
\text { Potion for Code Warriors. Hawaii International Conference on System Sciences, p.466-466, 7-10, } \\
\text { 2008. DOI: 10.1109/HICSS.2008.384 }\end{array}$ \\
\hline S0574 & $\begin{array}{l}\text { ŁUKASIEWICZ, K.; MILER, J. Improving agility and discipline of software development with the } \\
\text { Scrum and CMMI. Software, IET , vol.6, no.5, pp.416-422. 2012. DOI: } 10.1049 \text { /iet-sen.2011.0193 }\end{array}$ \\
\hline S0713 & $\begin{array}{l}\text { SALINAS, C. J. T; ESCALONA, M. J.; MEJIAS, M. A scrum-based approach to CMMI maturity } \\
\text { level } 2 \text { in web development environments. ii WAS-International Conference on Information } \\
\text { Integration and Web-based Applications \& Services. 2012. DOI:10.1145/2428736.2428782 }\end{array}$ \\
\hline S1026 & $\begin{array}{l}\text { NAWROCKI, J.R., WALTER, B.; WOJCIECHOWSKI A. Comparison of CMM level } 2 \text { and } \\
\text { eXtreme programming. 7th European Conference on Software Quality. } 2002 \text {. }\end{array}$ \\
\hline S1455 & $\begin{array}{l}\text { GARZÁS, J.; PAULK, M. C. A case study of software process improvement with CMMI-DEV and } \\
\text { Scrum in Spanish companies". Journal of Software: Evolution and Process. DOI: }\end{array}$ \\
\hline
\end{tabular}




\begin{tabular}{cl}
\hline ID & \multicolumn{1}{c}{ Referência } \\
\hline S2154 & http://dx.doi.org/10.1002/smr.1605 \\
S2195 & $\begin{array}{l}\text { BOUGRon, P.E. CMMI the agile way in constrained and regulated environments.(2016).Crosstalk. } \\
\text { of the third level of CMMI model in agile methods: Scrum, XP and Kanban. Colloquium in }\end{array}$ \\
& Information Science and Technology. CIST. 2015.
\end{tabular}

\subsection{Metodologias ágeis no contexto de aquisição de produtos e serviços dentro do SAM do CMMI_DEV}

Navarreteet al. [S0004] realizaram um estudo sobre a seleção de componentes de software de prateleira. Os autores fizeram um mapeamento teórico das práticas ágeis em relação ao processo de seleção de componentes no contexto do CMMI nível 2, propondo um framework chamado agileXcmmi, cruzando as áreas de processo do nível 2 do CMMI com as práticas ágeis que as suportam, em relação à seleção de componentes de software. No entanto, o estudo não apresenta dados primários empíricos ou práticos sobre o uso das práticas ágeis no contexto da gestão do acordo de fornecedores. Apenas limita-se a dados apresentados na literatura e à proposta de um framework teórico sobre a seleção de componentes de software sem validação prática na indústria.

Lee et al. [S0329] desenvolveram uma pesquisa onde descrevem como juntar metodologias ágeis e CMMI. Os autores identificaram e definiram um processo, mapeando de forma teórica as práticas de XP em relação ao CMMI níveis 2 e 3 a partir da comparação dos dados existentes na literatura. O estudo limita-se a dados teóricos e sem validação empírica na indústria.

Pikkarainen e Mäntyniemi [S0383] realizaram um mapeamento das práticas do Scrum e XP em relação aos objetivos específicos do CMMI níveis 2 e 3 . Os resultados mostraram a compatibilidade entre as abordagens em relação às áreas de gerenciamento de requisitos e gestão de projetos, porém deixaram claro que a área de processo de SAM não entrou no escopo do estudo.

Marcalet al. [S0449] apresentaram um mapeamento entre as áreas de processo do CMMI e as práticas da metodologia ágil Scrum. Eles mostraram como o Scrum trata as áreas de processo de gerenciamento de projetos do CMMI. O estudo limita-se a um mapeamento teórico, sem evidências empíricas, mostrando também que área de processo de SAM do CMMI não é atendida pelas práticas do Scrum.

Sutherland et al. [S0468] afirmam que o CMMI e o Scrum podem ser combinados com sucesso. No estudo conduzido foi proposto um guia através de um mapeamento das práticas ágeis do Scrum e Lean em relação aos objetivos e práticas do CMMI nível 5. Os resultados mostraram que o desempenho das equipes melhorou significativamente, mantendo a conformidade com CMMI nível 5, em comparação com o desempenho do CMMI, Scrum e Lean executados isoladamente. No entanto, o mapeamento não inclui as práticas e objetivos da área de processo de SAM.

Łukasiewicz e Miler [S0574] apresentaram um método combinando o Scrum com o modelo de maturidade CMMI para melhorar a agilidade e a disciplina do desenvolvimento de software. O estudo propôs um modelo de referência CMMI - Scrum, que mapeou as práticas do Scrum em relação às 123 práticas do CMMI nos níveis 2 e 3 . Os resultados mostraram a compatibilidade entre a maioria das práticas, sendo $24,5 \%$ incompatíveis e 3,5\% rejeitadas. O estudo deixou claro que não estudou as práticas da área de processo de SAM.

Salinas et al. [S0713] analisaram os objetivos do nível 2 de maturidade do CMMI e a viabilidade de alcançá-los usando as práticas propostas pelo Scrum, tentando avaliar se a utilização desta metodologia é adequada ou não para alcançar as metas genéricas e específicas do CMMI. Os resultados apontaram que uma abordagem CMMI nível 2 baseada exclusivamente em Scrum é insuficiente para alcançar todos os objetivos deste nível. O mapeamento mostrou que as práticas do Scrum não atendem os objetivos genéricos e específicos da área de processo de SAM do CMMI.

Nawrocki et al. [S1026] realizaram uma comparação entre o CMMI nível 2 e o XP. Os autores deixam claro que a área de processo de SAM do CMMI ficou fora do escopo da pesquisa.

Garzas e Paulk [S1455] desenvolveram um estudo de caso mostrando a relação entre o nível 2 do CMMIDEV 1.3 e o Scrum. O objetivo foi avaliar como o Scrum ajuda a implementar um modelo de processo, tal como CMMI-DEV. Um estudo de caso detalhado foi realizado em empresas de Tecnologia da Informação na Espanha. 
Os resultados mostraram que a maior parte das áreas de processo do CMMI-DEV nível 2 tinha sido melhorada pela utilização de Scrum, porém afirmam que a área de processo de SAM ficou fora do escopo do estudo.

McMahon [S2154] desenvolveu um estudo de caso em uma organização que reconheceu que tinham ido demasiado longe a abandonar seus processos CMMI em favor de uma aproximação ágil. $\mathrm{O}$ artigo descreve como a organização colocou rapidamente práticas do CMMI complementando sua abordagem ágil. Os resultados apontam que melhoraram o desempenho em apenas alguns meses usando uma combinação de três estruturas: CMMI, Scrum e Essência. No entanto, o estudo se limita a dizer que a gestão de fornecedores pode ser feita com práticas comuns do scrum, sem apresentar dados empíricos.

Bougroun et al. [S2195] apresentaram um mapeamento das práticas do CMMI em relação as práticas dos métodos ágeis Scrum, XP e Kanban. Os autores concluíram que a fusão dos três métodos cobre $58 \%$ da qualidade do processo do nível 3 do modelo CMMI três. Porém, os autores não analisaram a gestão de fornecedores, pois esta está no nível 2 do CMMI.

Os estudos mostraram que a gestão do acordo de fornecedores dentro do processo de aquisição por subcontratação no contexto do CMMI-DEV tem ficado de fora do escopo das pesquisas e consequentemente a não implementação das práticas de SAM do CMMI-DEV por estes. Esse fato, também pode ser observado nas avaliações do CMMI Institute [1], onde a área de Gestão do Acordo do Fornecedor do CMMI-DEV é muita das vezes excluída das avaliações realizadas nas empresas, por não fazer parte do contexto das unidades organizacionais em implementar as práticas constantes neste processo, e por isso, pouco se tem de relato de experiência sobre este assunto [1], conforme descoberto nesta revisão. O que não quer dizer que tal área (SAM_AGILE) não seja importante ou relevante no contexto de desenvolvimento de software, uma vez que trata da gestão dos fornecedores dos produtos, e pode-se notar a importância e a grande quantidade de aquisições feitas pelo Governo nestes últimos anos [52], seja em software ou serviços correlatos.

Mesmo sem encontrar estudos empíricos que tratam especificamente sobre SAM e metodologias ágeis, é fácil perceber que as metodologias ágeis podem agregar valor $[31,32,33,34,35]$ nesta gestão de fornecedores pelos próprios valores e princípios $[17,18]$ que neste cenário pode agregar para solucionar inúmeros problemas encontrados e relatados na literatura como: negligência em tratar os problemas; falta de código de conduta para fornecedores; falta de auditorias como parte de uma política de governança; falta de um canal aberto para que colaboradores, funcionários e parceiros "denunciem" possíveis riscos; falta de uma clareza na comunicação sobre quem são os fornecedores e quais as atividades eles realizam; escopo fechado; tempo fixo de desenvolvimento $[4,5,6,7,10,13,14,15]$. Assim, uma pesquisa que investigue como metodologias ágeis podem se inserir no contexto de gestão do acordo de fornecedor é relevante para resolver esses e outros problemas na relação existente entre cliente-fornecedor.

Diante do exposto, observa-se que a grande maioria dos estudos preocupou-se em mapear as práticas ágeis e algumas áreas de processo do CMMI de forma teórica, deixando claro que a área de processo de SAM do CMMI-DEV ficou fora do escopo dessas pesquisas. Os resultados encontrados apontam uma escassez de pesquisas sobre o tema, pois não revelaram dados relevantes sobre a combinação da área de processo de SAM do CMMI-DEV com as metodologias ágeis. No entanto, confirmam a lacuna de pesquisa na área investigada, justificando a relevância, viabilidade e o caráter inovador desta revisão, além de motivar estudos futuros mais detalhados baseados em métodos experimentais dentro das organizações, objeto de continuação desta pesquisa, visando à construção e a validação de um processo específico SAM_AGILE.

\subsection{Visão geral dos estudos}

Esta revisão buscou investigar a relação das principais metodologias ágeis com a área de processo de SAM do CMMI-DEV. Os resultados apontaram que o Scrum foi à metodologia mais utilizada em $54 \%$ dos projetos. A grande utilização do uso do Scrum pode ter sido motivada pelo fato do Scrum e do CMMI possuírem forte relação com a gestão de projetos, justificando sua escolha nos projetos. Esses dados estão em consonância com os achados das pesquisas em [20,21, 22,23]. A distribuição temporal dos 11 estudos mostra que entre 2003 e 2016 houve poucos estudos primários relacionados sobre gestão de fornecedores. Também mostra que uma pequena quantidade e distribuição irregular dos estudos ao longo dos anos não revelaram uma tendência crescente ou decrescente em relação à distribuição. A distribuição dos estudos por conferências e periódicos apontou que oito dos onze estudos foram publicados em conferências e três foram publicados em periódicos. Não foi possível destacar um veículo de publicação em particular, pois cada conferência ou periódico publicou 
apenas um estudo. A Tabela 5 apresenta a distribuição dos estudos considerando as bases de dados digitais como fonte de publicação. A Scopus foi o destaque com quatro publicações, seguido da IEEE com três.

Tabela 5: Distribuição dos Estudos por Base de Dados.

\begin{tabular}{ccc}
\hline ID & Quantidade & Fonte \\
\hline S0004 & 01 & Compendex (Springer) \\
S0329, S0383, S2154 S2195 & 04 & Scopus \\
S0449, S0468, S0574 & 03 & IEEE \\
S0713 & 01 & ACM Digital \\
S1026 & 01 & Springer \\
S1455 & 01 & Wiley Online Library \\
\hline
\end{tabular}

Em relação ao contexto dos estudos, a Tabela 6 mostra o tipo de estudo (industrial ou acadêmico), método de pesquisa, coleta dos dados, análise dos dados e o tamanho da amostra. A maioria possui dados empíricos com pesquisas realizadas na indústria. Em relação aos métodos de pesquisa, foram identificados três métodos de pesquisa: Estudo de caso, Experimento e Revisão. O estudo de caso foi o destaque, representado 5 estudos. O survey foi o instrumento de coleta de dados mais utilizado. A maioria dos estudos apresenta uma análise qualitativa dos dados. Esta revisão identificou 28 autores que contribuíram com essa temática. Também foram identificados os principais países dos autores envolvidos. No total foram 7 países envolvidos, com destaque para: Espanha, com 4 estudos. Destaca-se que foi identificada a participação do Brasil com 1 estudo, isso indica a necessidade de mais estudos nessa temática no país. Em relação às instituições de ensino e organizações públicas ou privadas foram identificadas 175 instituições envolvidas nos estudos. Devido a pouca quantidade de estudos não foi possível destacar uma instituição em particular.

\begin{tabular}{cccccc}
\multicolumn{5}{c}{ Tabela 6: Dados de contexto } \\
\hline ID & Tipo do estudo & $\begin{array}{c}\text { Método de } \\
\text { pesquisa }\end{array}$ & Coleta de dados & Análise dos dados & Amostra \\
& & & & Qualitativa & - \\
S0004 & Acadêmico & Revisão & Literatura & Qualitativa & - \\
S0329 & Acadêmico & Revisão & Literatura & Qualitativa & 7 projetos \\
S0383 & Industrial & Estudo de caso & Survey / Entrevista & Qualitativa & - \\
S0449 & Acadêmico & Revisão & Literatura & Qualitativa & 2 projetos \\
S0468 & Industrial & Estudo de caso & - & Quantitativa /qualitativa & 1 projeto \\
S0574 & Industrial & Estudo de caso & Survey & Qualitativa & - \\
S0713 & Acadêmico & Revisão & Literatura & Quantitativa/qualitativa & 6 projetos \\
S1026 & Industrial & Experimento & - & Quantitativa/qualitativa & 12 projetos \\
S1455 & Industrial & Estudo de caso & Survey/ Entrevista & Qualitativa & 1 projeto \\
S2154 & Industrial & Estudo de caso & Survey/ Entrevista & Qualitativa & - \\
S2195 & Acadêmico & Revisão & Literatura & & \\
\hline
\end{tabular}

\subsection{Limitações e ameaças à validade da pesquisa}

As principais características do protocolo, limitações e ameaças à validade que possam ter influenciado os resultados deste estudo, de acordo com Kitchenham e Charters [42], foram: (1) possíveis erros de classificação; (2) seleção apenas dos estudos num determinado período de tempo específico; (3) viés de seleção dos estudos; (4) credibilidade dos resultados; e (5) dificuldade de transferências dos resultados. No intuito de mitigar essas limitações e ameaças, as seguintes ações foram executadas: (1) realizou-se a seleção com dois pesquisadores e três revisores em cada fase da revisão, sendo que os conflitos foram resolvidos em reunião entre os pesquisadores; (2) buscou-se o maior período de tempo possível para minimizar o viés de período de busca; (3) para evitar o viés na seleção dos estudos, reuniões e guias foram conduzidos em cada etapa da pesquisa e estas foram conduzidas por dois pesquisadores; (4) para aumentar a credibilidade dos resultados, buscou-se a utilização de múltiplas fontes de dados como as principais conferências e periódicos na área de engenharia de software citados por Kitchenham et al. [48], especificamente relacionados com CMMI e Metodologias ágeis; (5) para aumentar a possibilidade de transferência dos resultados para outros contextos, o protocolo foi descrito por completo em detalhes. 


\section{Conclusões e trabalhos futuros}

O principal objetivo deste estudo foi explorar a literatura, a partir de um estudo de revisão sistemática, para analisar o atual estado da investigação sobre a relação entre Metodologias Ágeis e CMMI-DEV, no contexto da gestão do acordo de fornecedores. Os resultados não apresentaram evidências empíricas ou aplicações práticas do uso das metodologias ágeis no contexto da área de processo de SAM do CMMI-DEV, no entanto, esta revisão sugere que modelos de qualidade como CMMI e metodologias ágeis podem ser combinados com sucesso [S0004, S0329, S0383, S0449, S0468, S0574, S0713, S1026, S1455, S2154, S2195] para solução de problemas no contexto do processo de aquisição dentro do desenvolvimento de software. Os resultados também apontaram a viabilidade dessa junção, pois essas abordagens compartilham princípios e valores de gestão que podem contribuir para a melhoria do processo. Por outro lado, pode-se concluir que a gestão do acordo de fornecedores dentro do processo de aquisição no desenvolvimento de software no contexto do CMMIDEV e das metodologias ágeis tem sido pouco explorada de forma empírica, principalmente no sentido mais amplo, de combinar práticas de várias metodologias ágeis na busca pela melhoria da qualidade do processo de desenvolvimento e do produto. Esse fato reforça a necessidade de entender essa relação de forma detalhada, mostrando a necessidade de mais pesquisas na indústria.

Partindo dessa motivação, sugerem-se os seguintes trabalhos futuros: (1) criação de um framework genérico sobre a gestão do acordo de fornecedores baseada em práticas ágeis e modelos de qualidade, com o objetivo de mitigar os problemas mencionados, bem como, apoiar os pesquisadores e as empresas na definição, planejamento, execução e gestão da aquisição de produtos ou serviços nesse contexto; (2) desenvolvimento de estudos exploratórios como experimentos, pesquisa ação e survey para validar a aplicabilidade e o comportamento do framework SAM_AGILE nas empresas.

\section{Referências}

[1] CMMI INSTITUTE. Published Appraisal Results List. $2016 . \quad$ Disponível em: $<$ https://sas.cmmiinstitute.com/pars/pars.aspx>.Acesso em: 30 Julho 2016.

[2] PMI. A Guide to the Project Management Body of Knowledge (PMBOK). 2013.

[3] SOMMERVILLE, I. Engenharia de Software. 8. Ed, São Paulo: Pearson, 2007.

[4] SÖKMEN, N. Turkish Software Producing ICT Companies Approaches in Establishment of Their Subcontractor Selection and Management Processes. PICMET 2009 Proceedings, USA: Portland, 2009.

[5] SIKANDAR, A.; SIFFAT ULLAH, K. Software outsourcing partnership model: An evaluation framework for vendor organizations. Journal of Systems and Software, Volume 117, ISSN 0164-1212, 2016.

[6] JABANGWE R.; ŠMITE, D.; HESSBO, E. Distributed software development in an offshore outsourcing project: A case study of source code evolution and quality. Information and Software Technology, Volume 72, April 2016, Pages 125-136, ISSN 0950-5849, Disponível em: <https://doi.org/10.1016/j.infsof.2015.12.005>. Acesso em: 30 Junho 2017.

[7] FILIP, F. C.; KLEIN, V. M. Theoretical Concepts on Supplier Agreement. Management, Transilvania University of Brasov, p. 41-46, 2012.

[8] YING, C.; FUGUAN, S.; TINGBIN, C. Promotion of offshore software outsourcing enterprise value chain based on knowledge transfer effects.4th International Conference on Wireless Communication, Networking, 2008.

[9] BARDHAN, I.; MITHAS, S.; LIN S. Performance Impacts of Strategy, Information Technology Applications, and Business Process Outsourcing in U.S. Manufacturing Plants. Production and Operations Management. Vol. 16, No. 6. 2007, pp. 747-762. DOI: 10.3401/poms.

[10] CALVO-MANZANO, J. A.; SAN FELIU, T.; PURSCHE, A. The forgotten practices of subcontracting. Information Systems and Technologies (CISTI), p.1-4, 2010.

[11] WEBER, K.; ARAUJO, E. E. R.; SCAlET, D.; ANDRADE, E. L. P.; ROCHA, A. R. C.; MONTONI, M. A.MPS Model-Based Software Acquisition Process Improvement in Brazil. Sixth International Conference 
on the Quality of Information and Communications Technology, IEEE Computer Society, p. 110-119, 2007.

[12] VENKATRAMAN, N.; LOHL, L. Diffusion of Information Technology Outsourcing: Influence Sources and the Kodak Effect. Information Systems Research, 1995.

[13] KOMMEREN, R.; PARVIAINEN, P. Philips experiences in global distributed software development. Empirical Software Engineering, Volume 12, Number 6, 2007.

[14] HERBSLEB, J. D.; PAULISH, D., MATTHEW, B. J. Global Software Development at Siemens: Experience from Nine Projects.27th international conference on Software engineering, USA, 2005.

[15] BOSCH, J. Software Product Families in Nokia. In: Software Product Lines, 2005.

[16] SEI. CMMI para Desenvolvimento: Melhoria de processos visando melhores produtos e serviços (CMMI$D E V)$. versão 1.3. Ed, Pittsburgh, 2010.

[17] BeCK, K.; BeEDle, M.; BenNeKUM, A. V. Manifesto para Desenvolvimento Ágil de Software. 2001.Disponível em: <http://www.manifestoagil.com.br>. Acesso em: 30 Julho 2016.

[18] HIGHSMITH, J. Agile project management—creating innovative products. Boston, 2004.

[19] DYBÅ, T.; DINGSØYR, T. Empirical Studies of Agile Software Development: A Systematic Review. Information and Software Technology, Butterworth-Heinemann Newton, USA, v. 50, n. 9, 2008.

[20] VERSIONONE.6th Annual State of Agile Development Survey Results. 2011.

[21] VERSIONONE.7th Annual State of Agile Development Survey Results. 2012.

[22] VERSIONONE.8th Annual State of Agile Development Survey Results. 2014.

[23] VERSIONONE.9th Annual State of Agile Development Survey Results. 2015.

[24] SCHWABER, K.; SUTHERLAND, J. Guia do Scrum: um guia definitivo para o Scrum: as regras do jogo. 2011.

[25] BECK, K. Extreme Programming Explained: Embrace Change. ISBN 0-201-61641-6, 2000.

[26] BECK, K. Extreme Programming Explained: Embrace Change. 2. ed. ISBN 978-0321278654, 2004.

[27] COCKBURN, A. Crystal Clear: A Human-Powered Methodology for Small Teams. ISBN 0-201-69947-8, 2004.

[28] POPPENDIECK, M.; POPPENDIECK, T. Lean Software Development - An Agile Toolkit for Software Development Managers. Addison-Wesley, Boston, ISBN 0-321-15078-3, 2003.

[29] PALMER, S. R.; FELSING, J. M.A Practical Guide to Feature-driven-Development. Prentice Hall, Upper Saddle River, NJ, ISBN 0-13-067615-2, 2002.

[30] STAPLETON, J. DSDM: Business Focused Development. second ed., Pearson Education, ISBN 978$0321112248,2003$.

[31] PETERSEN, K.; WOHLIN, C. A. Comparison of Issues and Advantages in Agile and Incremental Development Between State of the Art and an Industrial Case. Journal of Systems and Software. 2009.

[32] HONG, N.; YOO, J.; CHA, S. Customization of Scrum Methodology for Outsourced Ecommerce Projects. Asia-Pacific Software Engineering Conference, 2010.

[33] CAO, L.;MOHAN, K.; XU, P.; RAMESH, B. Framework for Adapting Agile Development Methodologies. European Journal of Information Systems, Volume 18, No. 4, p. 332-343, 2010.

[34] MOE, N. B.;DINGSØYR, T.; DYBÅ, T.A Team work Model for Understanding an Agile Team: A Case Study of a Scrum Project. Information and Software Technology, Volume 52, no. 5, p. 480-491, 2010.

[35] LEE, S.; YONG, H. S. Distributed Agile: Project Management in a Global Environment. Empirical Software Engineering. Volume 15, No. 2, p. 204-217, 2010. 
[36] SANTOS, M.; MARQUES, A.; VACONCElOS, A.; OlIVEIRA, S.; KAMEI, F.; RODRIGUES, A.; SANTANA, D. Modeling The Impact Of Scrum And Xp In The Satisfaction Of Stakeholders Based On Thematic Analysis: A Systematic Study. In: 13th CONTECSI - International Conference on Information Systems and Technology Management, DOI: 10.5748/9788599693124-13CONTECSI/PS-3904, ISSN 2448-1041, 2016.

[37] SANTANA, C.; VASCONCElOS, A.; GUSMÃO, C. Software Process Improvement in Agile Software Development: A Systematic Literature Review. 41st Euromicro, 2015.

[38] SILVA F. S.; SOARES, F. S. F.; PERES, A. L.; AZEVEDO, I. M.; VASCONCELOS, A. P. L. F.; KAMEI, F. K.; MEIRA, S. R. L.Using CMMI together with agile software development: A systematic review. Information and Software Technology, 2015.

[39] DINGSØYR, T.; NERUR, S.; BALIJEPALLY, V.; MOE, N. B. A decade of agile methodologies: Towards explaining agile software development. Journal of Syst. and Soft., p. 1213-1221, 2012.

[40] PARSONS, D.; RYU, H.; LAL, R. The impact of methods and techniques on outcomes from agile software development projects In: McMASTER, Tom et al. (org.). Organizational Dynamics of Technology-Based Innovation: Diversifying the Research Agenda, Boston: Springer, p 235-249, 2007.

[41] EASTERBROOKS, S.; SINGER, J.; STOREY, M-A.; DAMIAN, D. Selecting Empirical Methods for Software Engineering Research. In: ASE 2007, Atlanta, Georgia, EUA, 2007.

[42] KITCHENHAM, B.; CHARTERS, S. Guidelines for performing Systematic Literature Reviews in Software Engineering. Keele University and Durham University Joint Report, EBSE 2007-001, 2007.

[43] MOJSILOVIC, A.; RAY, B.; LAWRENCE, R.; TAKRITI, S.A Logistic Regression framework for information technology outsourcing lifecycle management.Computers \& operations Research. 2007.

[44] NUNES, E. D.Definição de Processos de Aquisição de Software para Reutilização. Dissertação (Mestrado em Engenharia de Sistemas e Computação), Rio de Janeiro: UFRJ/COPPE, 2011.

[45] PATEL, C.; RAMACHANDRAN, M. Agile Maturity Model (AMM): A Software Process Improvement framework for Agile Software Development Practices. International Journal of Software Engineering, Vol.2, No.1, 2009.

[46] SANTANA, C.; GUSMÃO, C.; SOARES, L.; ROUILleR, A. C. Agile Software Development and CMMI: What We Do Not Know about Dancing with Elephants. Agile Processes in Software Engineering and Extreme Programming, XP 2009, Pula, Sardinia, Italy, 2009.

[47] JAKOBSEN, C.; JOHNSON, K. Mature Agile with a twist of CMMI. Agile Conference, 2008.

[48] KITCHENHAM, B.; DYBÅ, T.; JØRGENSEN, M. Evidence-based software engineering.26th International Conference on Software Engineering, (ICSE), IEEE, USA, p. 273 -281, 2004.

[49] LIKERT, R. A Technique for the Measurement of Attitudes. Archives of Psychology 140: p. 1-55, 1932.

[50] BEECHAN, S.; BADDOO, N.; HALL, T.; ROBINSON, H.; SHARP, H. Motivation in Software Engineering: A systematic literature review. Information and Software Technology: Elsevier, v. 50, n. 860 $878,2007$.

[51] CRUZES, D. S.; DYBÅ, T. Recommended Steps for Thematic Synthesis in Software Engineering. In: ESEM, 2011.

[52] COMPRASNET. Portal de Compras Governamentais. 2016. Disponível em: $<$ http://www.comprasgovernamentais.gov.br/>. Acesso em: 30 Julho 2016. 